\title{
Enfoque inicial del paciente estable con trauma precordial penetrante: ¿es tiempo de un cambio?
}

\author{
Conferencia "Rafael Casas Morales", XLIV Congreso Nacional \\ "Avances en Cirugía", Cartagena, Colombia, agosto de 2018.
}

\author{
Initial approach in the stable patient with penetrating precordial trauma. \\ Time for a change? \\ "Rafael Casas Morales" Honor lecture \\ Alberto García
}

Médico, cirujano de Trauma, Emergencias y Cuidado Intensivo; profesor asociado, Universidad del Valle, Cali, Colombia; profesor asociado, Universidad ICESI, Fundación Valle del Lili, Cali, Colombia

\section{Introducción}

Después de la introducción de la ventana pericárdica subxifoidea por Arom en $1977^{\text {I }}$, el enfoque inicial de los pacientes con trauma precordial penetrante se simplificó considerablemente, en la medida en que permitió evitar toracotomías innecesarias, único procedimiento diagnóstico disponible hasta ese momento. La aparición del examen por ultrasonido simplificó aún más el proceso diagnóstico ${ }^{2}$. Sin embargo, el reporte de falsos negativos, que pueden fluctuar alrededor del $15 \%{ }^{3,4}$ sumado a la falta de disponibilidad de los equipos, hacen que esta metodología no se aplique universalmente 5,6 .

Las técnicas mínimamente invasivas para practicar la ventana pericárdica ${ }^{7} \mathrm{y}$ la nueva interpretación del resultado positivo ${ }^{8,9}$, han hecho variar completamente el panorama y nos hacen pensar que es posible un cambio en el abordaje inicial de los pacientes con hemopericardio pero estables hemodinámicamente.

\section{Datos estadísticos}

Las lesiones ocasionadas por violencia interpersonal, a pesar de constituir un problema de salud pública en Colombia ${ }^{\mathrm{IO}, \mathrm{II}}$, brindan oportunidades de aprendizaje y mejoramiento continuo a los diferentes grupos quirúrgicos, por el considerable número de pacientes traumatizados que se atienden en nuestro país ${ }^{5-7,12-19}$.

Para mencionar algunos ejemplos, en I998, el I $7 \%$ de todas las muertes por trauma en Cali ocurrieron por traumatismos cardíacos. En ese año, la tasa de homicidios -solamente por trau-

Palabras clave: lesiones cardíacas; heridas penetrantes; historia; diagnóstico; ultrasonografía; técnicas de ventana pericárdica.

Key words: heart injuries; wounds, penetrating; history; diagnosis; ultrasonography; pericardial window techniques.

Fecha de recibido: 17/10/2018 - Fecha aceptación: 18/10/2018

Correspondencia: Alberto García, Calle 6 Oeste N 10-85, apartamento 202, torre 7, Cali, Colombia. Teléfono: (57) (315) 467-6870 Correo electrónico: alberto.garcia@correounivalle.edu.co

Citar como: García A. Enfoque inicial del paciente estable con trauma precordial penetrante: ¿es tiempo de un cambio? Rev Colomb Cir. 2019;34:16-24. https://doi.org/10.30944/20117582.93

Este es un artículo de acceso abierto bajo una Licencia Creative Commons - BY-NC-ND https://creativecommons.org/licenses/by-nc-nd/4.0/deed.es 
ma cardíaco- fue de I9 por I00.00o habitantes ${ }^{20}$ (tabla I). En 1995, Currea y Ferrada revisaron todos los traumas cardíacos que ocurrieron en Cali en 1993; identificaron 637 víctimas, de las cuales 217 llegaron vivas a los hospitales para ser atendidas; de estas, 136 egresaron vivas, para una supervivencia total del 2I \% y una supervivencia hospitalaria del $63 \%(\text { tabla } 2)^{13}$.

Sánchez, et al., reportaron una serie de 286 pacientes con trauma precordial penetrante, atendidos en el Hospital Universitario del Valle en 2003 y 2004, de los cuales 84 ingresaron con inestabilidad hemodinámica o con taponamien-

Tabla 1. Tasa de mortalidad por lesiones traumáticas, Cali, $1998^{20}$

\begin{tabular}{lcc}
\hline Estructura lesionada & Muertes & $\begin{array}{c}\text { Muertes por } \\
\mathbf{1 0 0 . 0 0 0 ^ { * }}\end{array}$ \\
\hline Mortalidad total & 2.145 & 112,0 \\
Trauma craneoencefálico & 1.153 & 60,2 \\
Corazón & 367 & 19,2 \\
Vasos pulmonares & 162 & 8,5 \\
Aorta torácica & 160 & 8,4 \\
Médula espinal & 134 & 7,0 \\
Hígado & 107 & 6,5 \\
\hline
\end{tabular}

* Población al 30 de junio de I998: I'915.43I

Extraído con permiso de: Garcia A, Lalsie R, Paredes J, Hurtado O, Ferrada R. Acta Colomb Cuid Intensivo.20oI;4:Io6 - 7 to cardiaco y fueron sometidos a toracotomía de emergencia. La mayoría de los 202 restantes fueron estudiados con una ventana pericárdica subxifoidea, la cual resultó positiva en $22 \%$ de los $\operatorname{casos}^{5}$ (figura I).

\section{Enfoque tradicional}

Hasta principios de los años ochenta, todos los pacientes con trauma precordial penetrante estables hemodinámicamente, eran estudiados por medio de una toracotomía exploratoria. Esto se asociaba con una gran incidencia de toracotomías innecesarias. En 1982, Garrison, et al. ${ }^{21}$, y, en 1984, Pérez, et al. ${ }^{22}$, propusieron estudiar este tipo de pacientes con una ventana pericárdica subxifoidea, lo cual disminuyó el número de to-

Tabla 2. Lugar del fallecimiento por trauma cardiaco penetrante, Cali, $1993^{15}$

\begin{tabular}{lcc}
\hline Lugar del fallecimiento & $\mathbf{n}$ & $\%$ \\
\hline Sitio del trauma & 254 & 50,7 \\
Durante el transporte & 166 & 33,1 \\
Hospital & 81 & 16,2 \\
Total & 501 & 100,0 \\
\hline $501(78,6 \%)$ muertos de 637 traumatizados &
\end{tabular}

$501(78,6 \%)$ muertos de 637 traumatizados

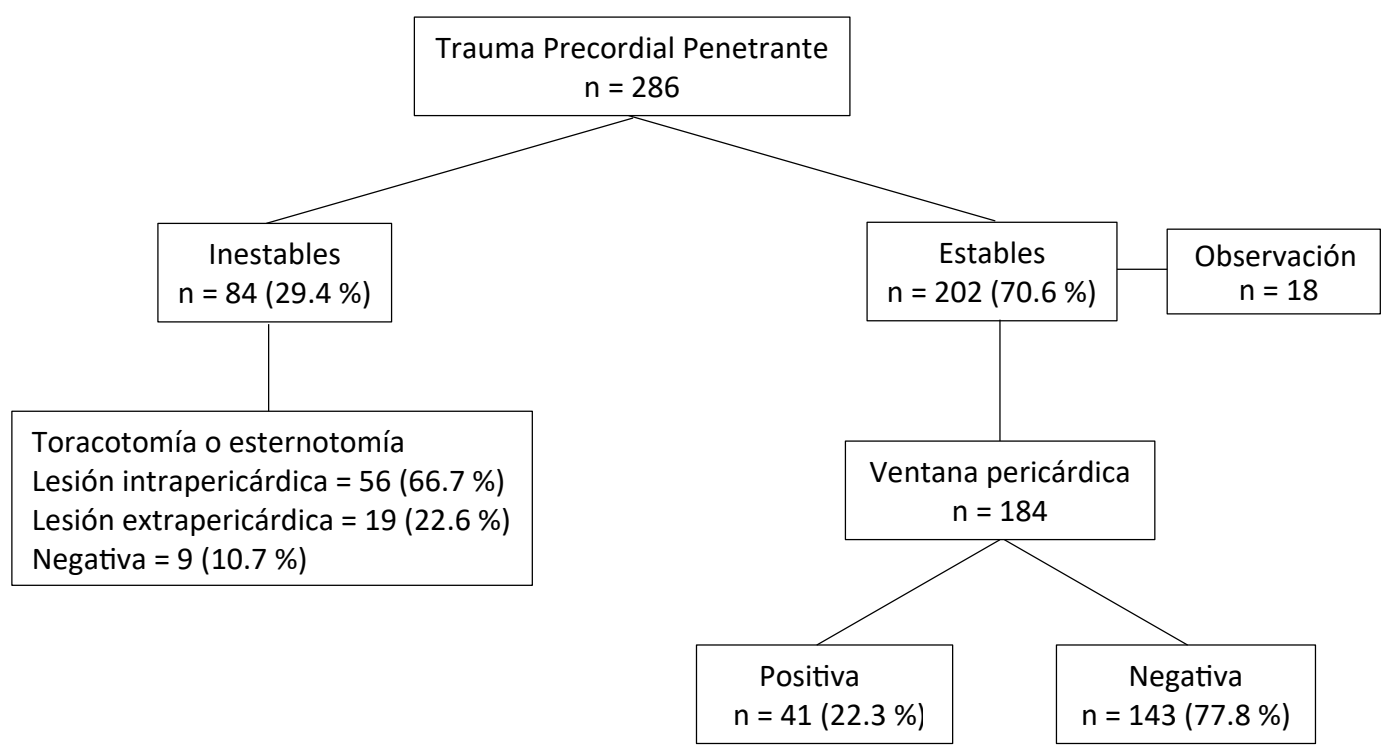

Figura 1. Enfoque inicial de los pacientes con trauma precordial penetrante. Hospital Universitario del Valle. 2003 - 2004. 
racotomías no terapéuticas de $58 \%$ a $5 \%$. Esta conducta fue aceptada rápidamente y se convirtió en el método de referencia, con una mediana de positividad para hemopericardio traumático del $22 \%^{1,5,7,13,21-29}$ (tabla 3).

En la figura I se ilustra el flujo de pacientes dentro de este esquema de manejo, reportado por Sánchez, et al. ${ }^{5}$. Se pudo comprobar que las dos terceras partes de los pacientes con trauma precordial que requirieron toracotomía, tuvieron como indicación la inestabilidad hemodinámica o el taponamiento cardiaco. La tercera parte restante fue detectada mediante una ventana pericárdica positiva.

Este enfoque resultaba bastante cómodo para los cirujanos y muy seguro para los pacientes, dado que la probabilidad de una lesión cardiaca inadvertida era prácticamente inexistente. Algunos grupos encontraron que sus inconvenientes eran la naturaleza invasiva de la ventana, la necesidad de anestesia general y el riesgo, aunque muy bajo, de complicaciones ${ }^{2,30-33}$.

En 1992, se informó por primera vez en Estados Unidos el uso del ultrasonido por cirujanos para el diagnóstico del trauma del torso ${ }^{34}$. En los siguientes años, se estandarizó la técnica de examen por ultrasonido para trauma de tórax y abdomen, y en I999, se publicó el estudio multicéntrico de Rozycki, et al., quienes reportaron

Tabla 3. Positividad de la ventana pericárdica para hemotórax traumático en los datos publicados

\begin{tabular}{|c|c|c|c|c|}
\hline Autor & Año & Técnica & $\mathbf{n}$ & $\begin{array}{c}\text { Positiva } \\
\text { n (\%) }\end{array}$ \\
\hline Andrade-Alegre ${ }^{23}$ & 1994 & Abierta & 75 & $17(22.7)$ \\
\hline Arom $^{1}$ & 1977 & Abierta & 50 & $16(32,0)$ \\
\hline Brewster ${ }^{24}$ & 1988 & Abierta & 108 & $30(27,8)$ \\
\hline Ferrada ${ }^{13}$ & 1997 & Abierta & 1101 & $235(21,3)$ \\
\hline Duncan ${ }^{25}$ & 1989 & Abierta & 51 & $12(23,5)$ \\
\hline Fraga ${ }^{26}$ & 2008 & Abierta & 207 & $39(18,8)$ \\
\hline Garrison ${ }^{21}$ & 1982 & Abierta & 108 & $19(17,6)$ \\
\hline Grewal ${ }^{27}$ & 1996 & Abierta & 122 & $26(21,3)$ \\
\hline Hommes ${ }^{28}$ & 2013 & Abierta & 50 & $14(28,0)$ \\
\hline Miller 29 & 1987 & Abierta & 104 & $19(18,3)$ \\
\hline Morales $^{7}$ & 1997 & Toracoscopia & 108 & $33(30,6)$ \\
\hline Pérez 22 & 1984 & Abierta & 70 & $18(25,4)$ \\
\hline Sánchez ${ }^{5}$ & 2016 & Abierta & 183 & $41(22,4)$ \\
\hline
\end{tabular}

una sensibilidad del Ioo \% y una especificidad del $97 \%{ }^{2}$. A pesar de que en este estudio no se hizo una prueba de control en todas las 225 ecografías negativas, su resultado fue ampliamente aceptado y, en los siguientes años, el ultrasonido reemplazó a la ventana pericárdica subxifoidea como método diagnóstico de referencia del hemopericardio traumático en los sujetos estables con trauma precordial penetrante.

Existen algunas limitaciones para la implementación del enfoque inicial de este grupo de pacientes, con base en la ecografía en la sala de emergencias. La primera es la falta de disponibilidad de equipos de ultrasonido en los lugares y a las horas requeridos. La segunda es la falta de entrenamiento en la técnica de ecografía de quienes hacen estas valoraciones, y la tercera es la falta de confianza en el examen por parte de algunos, debido al reporte de falsos negativos, que oscila entre el Io y el I5 \% 3,6, 19.

\section{Técnicas mínimamente invasivas}

En 1997, Morales, et al., reportaron una serie de Io8 pacientes con trauma precordial penetrante, estables, estudiados con una ventana pericárdica realizada por toracoscopia; en 3I \% de ellos se identificó hemopericardio traumático. Todos recibieron tratamiento quirúrgico abierto y ninguno presentó complicaciones secundarias al procedimiento ${ }^{7}$. Desde entonces, la ventana pericárdica por toracoscopia se ha incorporado al arsenal disponible para estudiar este grupo de pacientes.

A la fecha, se ha reportado la posibilidad de practicar ventanas pericárdica transdiafragmáticas por laparoscopia, y existen reportes ocasionales de reparo de lesiones cardiacas con técnicas mínimamente invasivas ${ }^{6,33,35-37}$ (figura 2).

\section{¿Se justifica una cirugía abierta en todos los hemopericardios traumáticos?} Hasta hace poco más de una década, el diagnóstico de hemopericardio de origen traumático estaba ligado a la necesidad de una toracotomía o de una esternotomía, con el objetivo de identificar y reparar la lesión cardiaca asociada. Diferentes 


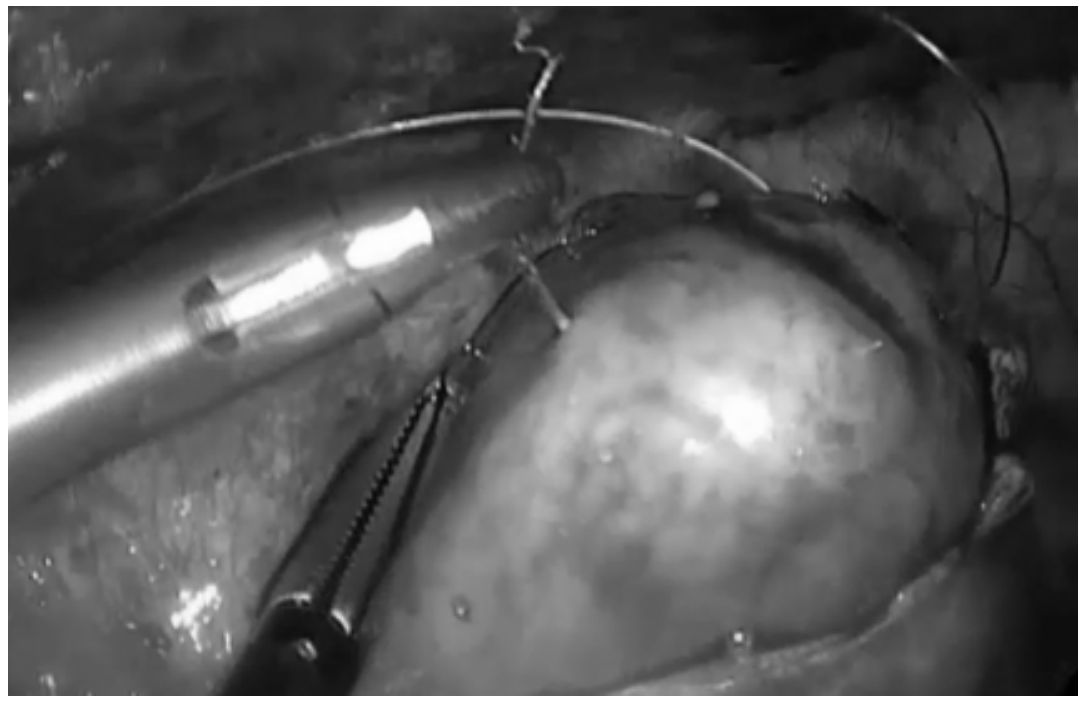

Figura 2. Reparo por toracoscopia de lesión de la cara anterior del ventrículo derecho. La ventana pericardica por toracoscopia había sido positiva y la lesión ventricular sangraba moderadamente (cortesía doctores Correa $\mathrm{J}$ y Zuluaga $\mathrm{M}$, Hospital Universitario del Valle)

autores han destacado en sus publicaciones la práctica de intervenciones no terapéuticas debido al hallazgo de una herida pericárdica o de una lesión tangencial de la musculatura cardiaca. En las publicaciones que incluyen más de 50 ventanas, se identificó una proporción de toracotomía o de esternotomía no terapéutica, después de una ventana positiva, entre el 6 y el $67 \%$, con una mediana de $23 \%$.

En 1995, Nagy, et al., propusieron la posibilidad de manejar los pacientes estables hemodinámicamente con ventana pericárdica subxifoidea positiva, con drenaje del pericardio y lavado. Reportaron cuatro pacientes manejados de esa manera de un grupo de 16 individuos con ventana pericárdica positiva ${ }^{38}$.

En 2005, Navsaria, et al., publicaron una serie de 2I pacientes estables con ventana pericárdica subxifoidea positiva. En I4 de ellos, se practicó una esternotomía después de verificar la estabilidad y la ausencia de sangrado, con el propósito de caracterizar las lesiones cardíacas existentes; se encontró una herida cardíaca de grado III o menos (compromiso pericárdico o tangencial del miocardio) en io de ellos. En los otros siete sujetos, se procedió a drenar el saco pericárdico, introduciendo una sonda para lavarlo y se instaló un drenaje pericárdico. No se practicaron esternotomías que, según lo establecido, debió haberse hecho. La evolución de los pacientes fue satisfactoria y no se detectaron complicaciones en el seguimiento ${ }^{8}$.

En 20I4, Nicol y Navsaria, de la Ciudad del Cabo, publicaron un ensayo clínico controlado aleatorizado, en el que incluyeron 111 pacientes estables después de ventana pericárdica subxifoidea positiva. Los 56 pacientes sometidos aleatoriamente a drenaje, lavado, instalación de drenaje y observación, evolucionaron sin requerir una intervención quirúrgica más compleja y sin mayor incidencia de complicaciones que los pacientes que fueron tratados con esternotomía. En 5I de los 55 pacientes asignados a cirugía abierta, se encontraron heridas pericárdicas o tangenciales. El curso posoperatorio de ambos grupos fue semejante ${ }^{39}$.

Además de los anteriores informes, se identificaron otros tres de pacientes manejados de esta manera (tabla 4) ${ }^{8,9,38-41}$. En el más reciente, González, et al., del Hospital Universitario del Valle en Cali, reportaron I2 pacientes manejados exitosamente de esta forma ${ }^{4 \mathrm{r}}$. 


\section{Experiencia local}

González, et al., y el grupo de la Universidad del Valle registraron prospectivamente los pacientes con trauma torácico atendidos desde septiembre de 2016 hasta enero de 2018 , e identificaron $\mathrm{I}_{4} 8$ individuos estables con trauma precordial penetrante, de los cuales II5 sufrieron heridas por arma corto-punzante.

Se practicó ventana pericárdica mediante técnicas mínimamente invasivas en 56 casos y, ventana pericárdica subxifoidea, en los 59 restantes. La ventana pericárdica fue positiva en 32

Tabla 4. Manejo conservador de los pacientes con ventana pericárdica positiva, series publicadas

\begin{tabular}{lcccc}
\hline Autor & Ciudad & Año & $\begin{array}{c}\text { Intervalo } \\
\text { (meses) }\end{array}$ & $\mathbf{n}$ \\
\hline Nagy $^{38}$ & Chicago & 1995 & 12 & 4 \\
Navsaria $^{8}$ & Ciudad del Cabo & 2005 & 6 & 7 \\
Thorson $^{9}$ & Miami & 2012 & SD & 3 \\
Nicol $^{39}$ & Ciudad del Cabo & 2014 & 87 & 56 \\
Chestovic $^{40}$ & Las Vegas & 2018 & 192 & 5 \\
González $^{41}$ & Cali & 2018 & 12 & 13 \\
\hline
\end{tabular}

SD: sin dato individuos, de los cuales I2 fueron manejados con evacuación del pericardio y lavado hasta descartar una hemorragia activa. Las dos terceras partes de los pacientes con ventana positiva abordados con técnicas mínimamente invasiva, fueron manejados sin toracotomía ni esternotomía; solo el $20 \%$ de los explorados con ventana pericárdica subxifoidea fue manejado de esta manera (tabla 5) ${ }^{4}$. La posibilidad de examinar la herida y comprobar por visualización directa la ausencia de sangrado, es responsable de esta diferencia (figuras 3 y 4 ).

Tabla 5. Manejo inicial de pacientes estables con trauma precordial, Hospital Universitario del Valle, noviembre de 2017 a diciembre de $2017^{41}$

\begin{tabular}{lccc}
\hline & $\begin{array}{c}\text { Abordaje } \\
\text { abierto }\end{array}$ & Cerrado & Total \\
\hline Total de pacientes & 92 & 56 & 148 \\
$\quad$ Ventana pericárdica negativa & 72 & 44 & 116 \\
$\quad \begin{array}{l}\text { Ventana pericárdica positiva } \\
\text { Tratamiento }\end{array}$ & 20 & 12 & 32 \\
$\quad \begin{array}{l}\text { Toracotomía o esternotomía } \\
\text { Conservador }\end{array}$ & 16 & 4 & 20 \\
& 4 & 8 & 12 \\
\hline
\end{tabular}
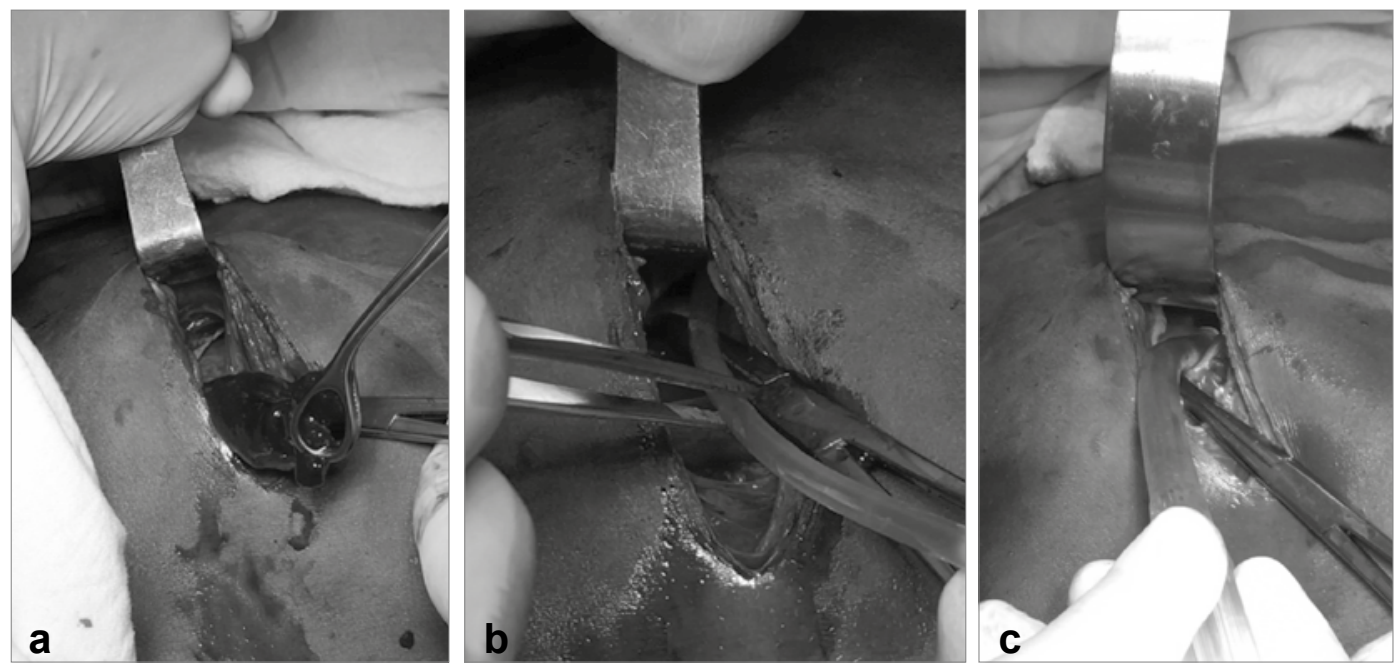

Figura 3. Manejo conservador de hemopericardio traumático, después de ventana pericardica subxifoidea positiva.

a. Evacuación del contenido del saco pericárdico, comprobando la ausencia de sangrado y la estabilidad hemodinámica.

b. Introducción de sonda al saco pericárdico para realizar lavado.

c. Aspiración de líquido cristalino al final del proceso de lavado. (cortesía doctor Adolfo González, Hospital Universitario del Valle). 

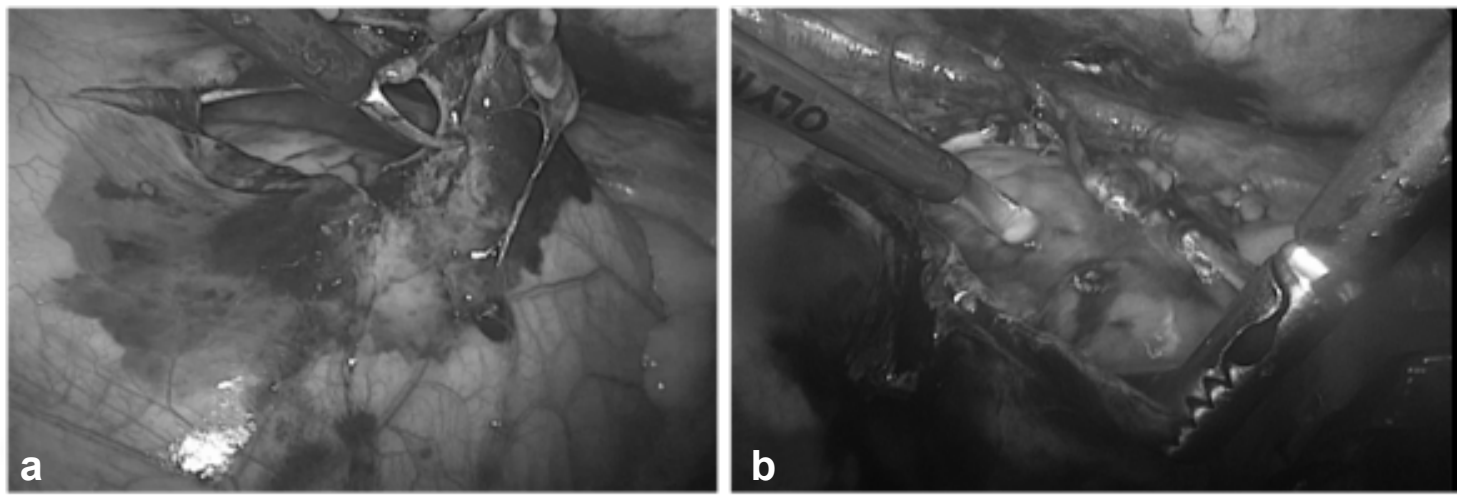

Figura 4. manejo conservador de hemopericardio traumático, mediante toracoscopia.

a. Ampliación de la ventana practicada previamente, después de comprobar la estabilidad y la ausencia de sangrado. b. Lavado de una herida superficial del ventrículo derecho, comprobando la ausencia de sangrado. (cortesía, doctor Alaín Herrera, Hospital Universitario del Valle)

\section{¿Es posible avanzar hacia un manejo no operatorio?}

En la Fundación Valle del Lili en Cali se ha avanzado hacia un manejo no operatorio con base en la observación clínica de los pacientes estables con derrame pericárdico documentado por ecografía.

De agosto de 2017 hasta julio de 20I8, el grupo de cirujanos de trauma de este hospital ha evaluado 45 pacientes clínicamente estables con trauma precordial. Se descartó la presencia de derrame pericárdico en la evaluación hecha con ultrasonido en 3I de ellos. Su evolución clínica no tuvo eventualidades.

De los I4 pacientes con derrame pericárdico identificado en la ecografía, cuatro recibieron observación clínica estrecha y seguimiento con ecografías diarias (figura 5). Ninguno presentó deterioro, requirió intervención quirúrgica o presentó complicaciones en el seguimiento a 28 días. Los io pacientes restantes fueron manejados con ventana pericárdica, la cual resultó negativa en cinco y positiva en los otros cinco. En cuatro de estos, se practicó una toracotomía o una esternotomía. El paciente restante fue tratado con drenaje y lavado del saco pericárdico y observación posoperatoria. En su evolución tampoco hubo complicaciones y no requirió intervención qui- rúrgica (Chica J, comunicación personal, Agosto de 2018) (figura 6).

\section{Conclusiones y direcciones futuras}

Con base en la información publicada y en las experiencias presentadas, se puede concluir lo siguiente.

I. Alrededor del $20 \%$ de los pacientes con trauma precordial penetrante, clínicamente estables, presentan un hemopericardio cuando el diagnóstico se hace por ventana pericárdica. Esta proporción se reduce de 30 a $50 \%$ cuando el diagnóstico es hecho con ultrasonido.

2. Más del $90 \%$ de este grupo presenta lesiones pericárdicas o miocárdicas tangenciales, la mayoría de ellas de resolución espontánea, aparentemente de poca relevancia clínica.

3. El abordaje diagnóstico mediante técnicas mínimamente invasivas es posible.

4. En pacientes estables hemodinámicamente con ventana pericárdica positiva, el tratamiento conservador con drenaje, irrigación y comprobación de ausencia del sangrado, se puede llevar a cabo dentro de un protocolo estricto de monitorización intraoperatoria y vigilancia posquirúrgica estrecha.

5. El manejo no operatorio de pacientes con trauma precordial penetrante, estables he- 
modinámicamente, con derrame pericárdico diagnosticado por ultrasonido, parece factible en un hospital con recursos para practicar la intervención quirúrgica de inmediato y bajo un protocolo de vigilancia estricta.

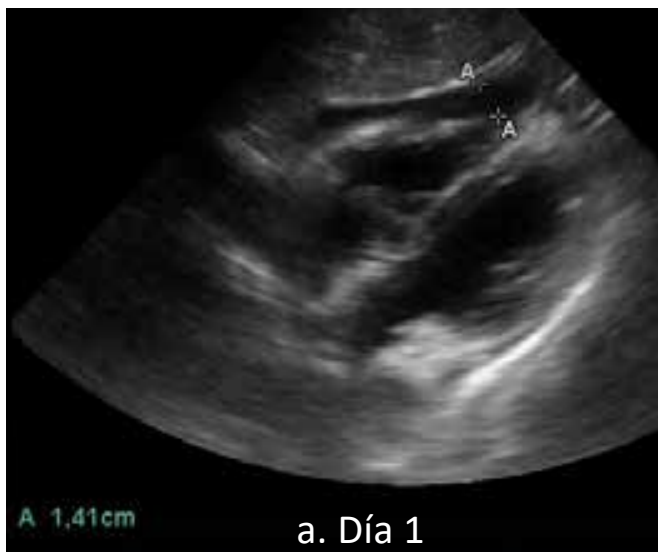

Estos avances orientados a la disminución del número de cirugías no terapéuticas y al uso óptimo de los recursos, se deben corroborar con estudios prospectivos en los que se incluyan pacientes de diferentes instituciones.

Figura 5. Seguimiento ecográfico de paciente estable hemodinámicamente.

a. Día primero. Derrame pericárdico que en su máxima dimensión mide 1.41 centímetros

b. Día sexto. Después de disminución progresiva, en el día sexto se encuentra una máxima distancia del derrame de $0.37 \mathrm{~cm}$.

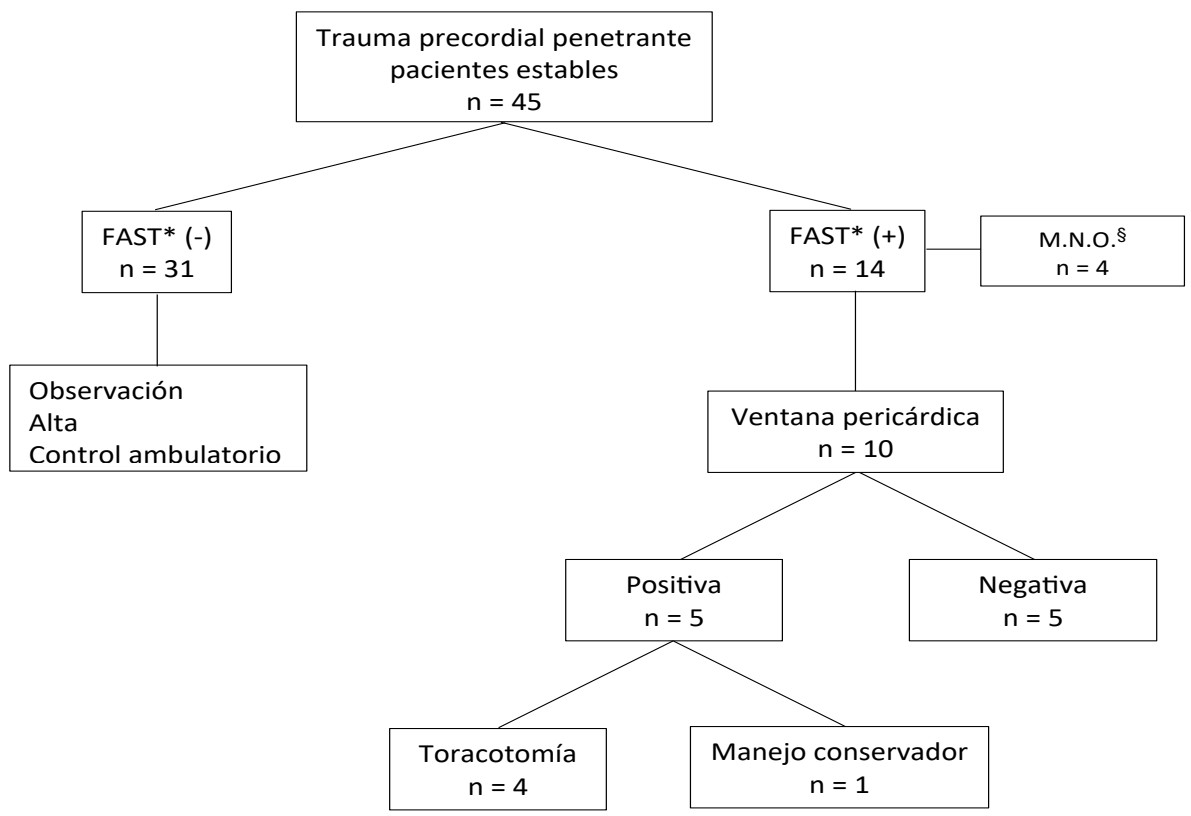

Figura 6. Enfoque inicial de los pacientes estables con trauma precordial penetrante. Fundación Valle del Lili. Agosto 2017 - Julio 2018.

"FAST: Ecografía enfocada en el diagnóstico de hemoperitoneo o hemopericardio en trauma.

$\$$ M.N.O. Manejo no operatorio 


\section{Referencias}

I. Arom KV, Richardson JD, Webb G, Grover FL, Trinkle JK. Subxiphoid pericardial window in patients with suspected traumatic pericardial tamponade. Ann Thorac Surg. 1977;23(6):545-9.

2. Rozycki GS, Feliciano DV, Ochsner MG, Knudson MM, Hoyt DB, Davis F, et al. The role of ultrasound in patients with possible penetrating cardiac wounds: a prospective multicenter study. J Trauma. 1999;46(4):5435I; discussion 5I-2.

3. Ball CG, Williams BH, Wyrzykowski AD, Nicholas JM, Rozycki GS, Feliciano DV. A caveat to the performance of pericardial ultrasound in patients with penetrating cardiac wounds. J Trauma. 2009;67(5):I123-4.

4. Nicol AJ, Navsaria PH, Beningfield S, Hommes M, Kahn D. Screening for occult penetrating cardiac injuries. Ann Surg. 20I5;26I(3):573-8.

5. Sanchez AI, Garcia A, Velásquez M, Puyana JC. Predictors of positive subxiphoid pericardial window in stable patients with penetrating injuries to the precordial region. Panam J Trauma. 20I6;5:43 - 5I.

6. Delgado-Mateus LJ, Bustos-Guerrero AM, BarcoManrique AG, Serrano-Pastrana JP, Herrán Falla OF, Valencia-Ángel LI. Ventana pericárdica: Abordaje subxifoideo vs toracoscópico en trauma precordial abierto. Panam J Trauma. 20I7;6(3):135 - 4I.

7. Morales CH, Salinas CM, Henao CA, Patino PA, Munoz CM. Thoracoscopic pericardial window and penetrating cardiac trauma. J Trauma. 1997;42(2):273-5.

8. Navsaria PH, Nicol AJ. Haemopericardium in stable patients after penetrating injury: is subxiphoid pericardial window and drainage enough? A prospective study. Injury. 2005;36(6):745-50.

9. Thorson CM, Namias N, Van Haren RM, Guarch GA, Ginzburg E, Salerno TA, et al. Does hemopericardium after chest trauma mandate sternotomy? J Trauma Acute Care Surg. 2012;72(6):I5I8-24; discussion 24-5.

Io. Acosta-Ramirez N, Peñaloza R, Garcia J. Carga de enfermedad en Colombia 2005: Resultados alcanzados. Bogota 2008. Descargado en www.javeriana.edu.co/ cendex/GPES/informes/PresentacionCarga_Informe. pdf, Julio de 2018

II. Marthe-Manjarrés A. Comportamiento de las lesiones por violencia interpersonal. Colombia, 20I7. Forensis Datos para la vida. 20I8;19(I):I24 - 70.

I2. Ferrada R, A G. Torso Penetrating Trauma. In: Maull K, Cleveland H, Feliciano D, Rice C, Trunkey D, Wolferth C, editors. Advances in trauma and Critical Care. Advances in trauma and Critical Care. 8. St Luis, MO: Mosby Year Book; 1993. p. 85- II6.

I3. Ferrada R. Penetrating Cardiac Trauma. Panam J Trauma. 2004;II(I):30 - 5 .
I4. Duque HA, Florez LE, Moreno A, Jurado H, Jaramillo CJ, Restrepo MC. Penetrating cardiac trauma: follow-up study including electrocardiography, echocardiography, and functional test. World J Surg. I999;23(I2):I254-7.

I5. Ferrada R, Rodriguez A. Trauma cardiaco. Tratamiento quirurgico. Rev Colomb Cir. 200I;I6(I):5 - I5.

I6. Gutiérrez M, Sánchez A, Muñoz E, Fandiño A, Muñoz E. Muertes ocurridas por lesiones de tránsito en el año 2004 2005. Consultado en: http://vigilesiones.univalle.edu.co/informes/anual/informestransito.html. Consultado en Julio de 2018

I7. Villegas MI, Morales-Uribe CH, Rosero-Cerón E, Benítez-España G, Cano-Restrepo F, Fernández IM, et al. Trauma cardíaco penetrante: factores pronósticos. Rev Colomb Cir. 2007;22(3):I48 - 56.

I8. Castano W, Morales CH, Senior JM, Benjumea WY, Sanchez J. Relationship of echocardiographic and coronary angiographic findings in patients with acute myocardial infarction secondary to penetrating cardiac trauma. J Trauma Acute Care Surg. 20I2;73(I):III-6.

19. Isaza-Restrepo A, Bolivar-Saenz DJ, Tarazona-Lara M, Tovar JR. Penetrating cardiac trauma: analysis of 240 cases from a hospital in Bogota, Colombia. World J Emerg Surg. 20I7;I2:26.

20. Garcia A, Lalsie R, Paredes J, Hurtado O, Ferrada R. Mortalidad prevenible por trauma en Cali. Colombia, 1998. Acta Col Cuidado Intensivo. 200I;4:I06 - 7 .

2I. Garrison RN, Richardson JD, Fry DE. Diagnostic transdiaphragmatic pericardiotomy in thoracoabdominal trauma. J Trauma. 1982;22(2):147-9.

22. Pérez M, Rubiano J, González A, Torres E, Kestenberg A. manejo de heridas precordiales en trauma precordial. Un nuevo enfoque. Colombia Medica. 1984;I5(4):I44 - 7 .

23. Andrade-Alegre R, Mon L. Subxiphoid pericardial window in the diagnosis of penetrating cardiac trauma. Ann Thorac Surg. 1994;58(4):I139-4I.

24. Brewster SA, Thirlby RC, Snyder WH, 3rd. Subxiphoid pericardial window and penetrating cardiac trauma. Arch Surg. I988;123(8):937-4I.

25. Duncan AO, Scalea TM, Sclafani SJ, Phillips TF, Bryan D, Atweh NA, et al. Evaluation of occult cardiac injuries using subxiphoid pericardial window. J Trauma. 1989;29(7):955-9; discussion 9-60.

26. Fraga GP, Espinola JP, Mantovani M. Pericardial window used in the diagnosis of cardiac injury. Acta Cir Bras. 2008;23(2):208-I5.

27. Grewal H, Ivatury RR, Divakar M, Simon RJ, Rohman M. Evaluation of subxiphoid pericardial window used in the detection of occult cardiac injury. Injury. I995;26(5):305-IO.

28. Hommes M, Nicol AJ, van der Stok J, Kodde I, Navsaria $\mathrm{PH}$. Subxiphoid pericardial window to exclude occult 
cardiac injury after penetrating thoracoabdominal trauma. Br J Surg. 20I3;IOO(II):I454-8.

29. Miller FB, Bond SJ, Shumate CR, Polk HC, Jr., Richardson JD. Diagnostic pericardial window. A safe alternative to exploratory thoracotomy for suspected heart injuries. Arch Surg. 1987;I22(5):605-9.

30. Yugueros P, Sarmiento JM, Ferrada R. Sindrome Postpericardiotomia por trauma. Rev Colomb Cir. 1993;8:I09 - I2.

3I. Jimenez E, Martin M, Krukenkamp I, Barrett J. Subxiphoid pericardiotomy versus echocardiography: a prospective evaluation of the diagnosis of occult penetrating cardiac injury. Surgery. 1990;108(4):676-9; discussion 9-80.

32. Meyer DM, Jessen ME, Grayburn PA. Use of echocardiography to detect occult cardiac injury after penetrating thoracic trauma: a prospective study. J Trauma. I995;39(5):902-7; discussion 7-9.

33. King DR, Vlahakes GJ, Johri AM, Sheikh AY. Postpericardiotomy syndrome from transdiaphragmatic pericardial window following trauma: first description and review of the literature. J Cardiovasc Med (Hagerstown). 2009;IO(IO):806-9.

34. Tso P, Rodriguez A, Cooper C, Militello P, Mirvis S, Badellino MM, et al. Sonography in blunt abdominal trauma: a preliminary progress report. J Trauma. I992;33(I):39-43; discussion -4.

35. O'Brien PK, Kucharczuk JC, Marshall MB, Friedberg JS, Chen Z, Kaiser LR, et al. Comparative study of subxiphoid versus video-thoracoscopic pericardial "window". Ann Thorac Surg. 2005;80(6):2013-9.
36. Navsaria PH, Nicol AJ. Video-assisted thoracoscopic pericardial window for penetrating cardiac trauma. S Afr J Surg. 2006;44(I):I8-20.

37. Correa Marin J, Zuluaga M, Urrea Llano JD. Positive video-assisted thoracoscopic pericardial window management of a right ventricle stab wound with minimally invasive technique. J Vis Surg. 20I6;2:IIo.

38. Nagy KK, Lohmann C, Kim DO, Barrett J. Role of echocardiography in the diagnosis of occult penetrating cardiac injury. J Trauma. 1995;38(6):859-62.

39. Nicol AJ, Navsaria PH, Hommes M, Ball CG, Edu S, Kahn D. Sternotomy or drainage for a hemopericardium after penetrating trauma: a randomized controlled trial. Ann Surg. 2014;259(3):438-42.

40. Chestovich PJ, McNicoll CF, Fraser DR, Patel PP, Kuhls DA, Clark E, et al. Selective use of pericardial window and drainage as sole treatment for hemopericardium from penetrating chest trauma. Trauma Surg Acute Care Open. 2018;3(I):eooor87.

4I. González-Hadad A, Osorno-Moncayo J, Ángel-Escobar I, Serna J, Salcedo A, Acero D, et al. Don't rush to a wide-open chest when diagnosing a hemopericardium in hemodynamically stable patients with penetrating injuries to the cardiac box. Presentado en el $77^{\circ}$ congreso de la Asociación Americana de Cirugía de Trauma, en San Diego, CA, Septiembre de 2018 [Disponible en: http://www.aast.org/AnnualMeeting/PastAbstracts.aspx. Descargado en Julio de 2018. 